\title{
The Semimartingale Approach to Almost Sure Stability Analysis of a Two-Stage Numerical Method for Stochastic Delay Differential Equation
}

\author{
Qian Guo and Xueyin Tao \\ Department of Mathematics, Shanghai Normal University, Shanghai 200234, China \\ Correspondence should be addressed to Qian Guo; qian_guo@hotmail.com
}

Received 16 June 2014; Accepted 11 September 2014; Published 23 October 2014

Academic Editor: Chengming Huang

Copyright (c) 2014 Q. Guo and X. Tao. This is an open access article distributed under the Creative Commons Attribution License, which permits unrestricted use, distribution, and reproduction in any medium, provided the original work is properly cited.

Almost sure exponential stability of the split-step backward Euler (SSBE) method applied to an Itô-type stochastic differential equation with time-varying delay is discussed by the techniques based on Doob-Mayer decomposition and semimartingale convergence theorem. Numerical experiments confirm the theoretical analysis.

\section{Introduction}

In this paper we study the following nonlinear SDDE:

$$
\begin{aligned}
\mathrm{d} X(t)= & f(X(t), X(t-\tau(t))) \mathrm{d} t \\
& +g(X(t), X(t-\tau(t))) \mathrm{d} W(t),
\end{aligned}
$$

for every $t \geq 0$. Here $\tau(t)$ is a time-varying delay satisfying $\tau>0$ and $-\widetilde{\tau}:=\inf \{t-\tau(t): t \geq 0\}$. The initial function $X(t)=\psi(t)$ when $t \in[-\tilde{\tau}, 0]$. We further assume that the initial data is independent of Wiener measure driving the equation and $W(t)$ is a scalar Brownian motion on the complete probability space $\left(\Omega, \mathscr{F}, \mathscr{F}_{t \geq 0}, \mathbb{P}\right)$ with a filtration satisfying the usual conditions. Moreover, $f, g: \mathbb{R}^{d} \times \mathbb{R}^{d} \rightarrow$ $\mathbb{R}^{d}$ are Borel-measurable functions.

Stability theory for numerical methods applied to stochastic differential equation (SDE) typically deals with mean-square behavior [1]. The mean-square stability analysis of numerical methods for SDDE has received a great deal of attention (see, e.g., [2, 3] and the references therein). Recently, the almost sure (a.s.) stability (or the trajectory stability) is becoming prevalent in the science literature [411]. However, the prior works concerned with SDDE are [7, 8, 10]. Rodkina et al. [7] studied almost sure stability of a driftimplicit $\theta$-method applied to an SDE with memory. Using the martingale techniques, $\mathrm{Wu}$ and his coauthors $[8,10]$ discussed almost sure exponential stability of the EulerMaruyama (EM) method for the SDE with a constant delay and stochastic functional differential equation. We note that the two above schemes are all single-stage method; this paper studies the almost sure stability of a two-stage scheme named split-step backward Euler (SSBE) method [12,13] applied to the nonlinear SDDE (1) with time-varying delay.

Applying the SSBE method (see $[12,13]$ ) to (1) yields

$$
\begin{gathered}
x_{n}^{*}=x_{n}+h f\left(x_{n}^{*}, \tilde{x}_{n}\right), \\
x_{n+1}=x_{n}^{*}+g\left(x_{n}^{*}, \tilde{x}_{n}\right) \Delta w_{n},
\end{gathered}
$$

where $\Delta w_{n}:=W\left(t_{n+1}\right)-W\left(t_{n}\right)$ and for $0 \leq \mu \leq 1, q_{n} \in \mathbb{Z}^{+}$,

$$
\begin{aligned}
& \tilde{x}_{n} \\
& = \begin{cases}\psi\left(t_{n}-\tau\left(t_{n}\right)\right), & t_{n}-\tau\left(t_{n}\right)<0 ; \\
\mu x_{n-q_{n}+1}+(1-\mu) x_{n-q_{n}}, & 0 \leq t_{n}-\tau\left(t_{n}\right) \\
& \in\left[t_{n-q_{n}}, t_{n-q_{n}+1}\right) .\end{cases}
\end{aligned}
$$

Here $h$ is the step size and $x_{n}$ denotes the approximation of $X(t)$ at time $t_{n}=n h(n=0,1, \ldots)$. We remark that $\mu$ in (3) depends on how memory values are handled on nongrid points. The almost sure convergence of SSBE method has been investigated by Guo and Tao [14]; the main aim of this paper is to study the almost sure stability of the SSBE method applied to (1). 


\section{Preliminary Results}

Before stating the main results, we present the essential notation and definitions which are necessary for further consideration. Let $|\cdot|$ be the Euclidean norm in $\mathbb{R}^{d}$ and $C\left([-\widetilde{\tau}, 0] ; \mathbb{R}^{d}\right)$ the family of continuous functions $\varphi$ from $[-\widetilde{\tau}, 0]$ to $\mathbb{R}^{d}$, equipped with the supremum norm $\|\varphi\|=$ $\sup _{-\tilde{\tau} \leq \theta \leq 0}|\varphi(\theta)|$. Also, denote by $C_{\mathscr{F}_{0}}^{b}\left([-\tilde{\tau}, 0] ; \mathbb{R}^{d}\right)$ the family of bounded, $\mathscr{F}_{0}$-measurable, $C\left([-\widetilde{\tau}, 0] ; \mathbb{R}^{d}\right)$-valued random variables. If $A$ is a vector or matrix, its transpose is denoted by $A^{T}$. The inner product of $X, Y \in \mathbb{R}^{d}$ is denoted by $\langle X, Y\rangle$ or $X^{T} Y$.

Now we give some definitions on the almost sure exponential stability of SDDEs and its numerical approximation.

Definition 1. The solution $X(t, \psi)$ to (1) is said to be almost surely exponentially stable if there exists a constant $\eta>0$ such that

$$
\limsup _{t \rightarrow \infty} \frac{1}{t} \log |X(t, \psi)| \leq-\eta \quad \text { a.s }
$$

for any initial data $\psi \in C_{\mathscr{F}_{0}}^{b}\left([-\widetilde{\tau}, 0] ; \mathbb{R}^{d}\right)$.

Definition 2. The solution $x_{n}$ to (2a) and (2b) is said to be almost surely exponentially stable if there exists a constant $\gamma>0$ such that

$$
\limsup _{n \rightarrow \infty} \frac{1}{n h} \log \left|x_{n}\right| \leq-\gamma \quad \text { a.s }
$$

for any bounded variables $\psi(k h)$ when $k h \in[-\tilde{\tau}, 0]$.

For the purpose of stability, we assume that $f(0,0, t)=$ $g(0,0, t)=0$, which implies that (1) admits the equilibrium solution $X(t)=0$ corresponding to the initial condition $\psi(t)=0$ for $t \in[-\widetilde{\tau}, 0]$. As a standing hypothesis, we will impose the following local Lipschitz condition (cf. [11, 12, 14]) on the coefficients $f$ and $g$.

(A1) For each integer $D$, there exists a positive constant $K_{D}$ such that, for all $x_{1}, x_{2}, y_{1}, y_{2} \in R^{d}$ with $\left|x_{1}\right| \vee\left|x_{2}\right| \vee$ $\left|y_{1}\right| \vee\left|y_{2}\right| \leq D$, and all $t \geq 0,\left|f\left(x_{1}, y_{1}\right)-f\left(x_{2}, y_{2}\right)\right|^{2} \vee$ $\left|g\left(x_{1}, y_{1}\right)-g\left(x_{2}, y_{2}\right)\right|^{2} \leq K_{D}\left(\left|x_{1}-x_{2}\right|^{2}+\left|y_{1}-y_{2}\right|^{2}\right)$, where $\mathrm{V}$ is the maximal operator.

To guarantee the almost sure stability of the unique solution to (1), we need the following assumption for the time-varying delay $\tau(t)$.

(A2) Let the delay function $\tau(t):[0,+\infty) \rightarrow[0, \bar{\tau}]$ be Borel measurable and bounded.

In what follows we introduce the result of almost sure stability of SDDEs (1). The proof of the following lemma can be found in [15].
Lemma 3. Let Assumptions (A1) and (A2) hold. Assume that there are four nonnegative constants $\lambda_{1}-\lambda_{4}$ such that

$$
\begin{gathered}
2 x^{T} f(x, 0) \leq-\lambda_{1}|x|^{2}, \\
|f(x, y)-f(x, 0)| \leq \lambda_{2}|y|, \\
|g(x, y)|^{2} \leq \lambda_{3}|x|^{2}+\lambda_{4}|y|^{2}
\end{gathered}
$$

for all $t \geq t_{0}$ and $x, y \in \mathbb{R}^{d}$. If

$$
\lambda_{1}>2 \lambda_{2}+\lambda_{3}+\lambda_{4}
$$

then the trivial solution of (1) is almost surely exponentially stable.

To explain our idea, we cite the discrete semimartingale convergence theorem as follows.

Theorem 4 (see $[8,9]$ ). Let $Z=\left(Z_{n}\right)_{n \in N}$ be an almost sure nonnegative stochastic sequence of $\left(\mathscr{F}_{n}, \mathscr{B}\right)$-measurable random variables $Z_{n}$ on probability space $\left(\Omega, \mathscr{F},\left(\mathscr{F}_{n}\right)_{n \in N}, \mathbb{P}\right)$. Assume that $Z$ permits the decomposition

$$
Z_{n} \leq Z_{0}+A_{n}^{1}-A_{n}^{2}+\mathscr{M}_{n}, \quad n \in N,
$$

where $A^{1}=\left(A_{n}^{1}\right)_{n \in N}$ and $A^{2}=\left(A_{n}^{2}\right)_{n \in N}$ are two nondecreasing, predictable processes with $A_{0}^{i}=0(i=1,2)$; $\mathscr{M}=\left(\mathscr{M}_{n}\right)_{n \in N}$ is local $\left(\mathscr{F}_{n}\right)_{n \in N^{-}}$martingale with $\mathscr{M}_{0}=0$ on $\left(\Omega, \mathscr{F},\left(\mathscr{F}_{n}\right)_{n \in N}, \mathbb{P}\right)$. Then, the requirement of $\lim _{n \rightarrow+\infty} A_{n}^{1}<$ $+\infty$ (a.s.) implies that

$$
\lim _{n \rightarrow+\infty} \sup Z_{n}<+\infty, \quad \lim _{n \rightarrow+\infty} A_{n}^{2}<+\infty
$$

for almost all $\omega \in \Omega$.

\section{Almost Sure Asymptotic Exponential Stability of Numerical Solution}

In this section, our aim is to examine if the SSBE method can reproduce the almost sure exponential stability of the exact solution of (1). Comparing to the existing results of singlestage methods $[8,10]$, we need to appropriately estimate the intermediate solution $x_{n}^{*}$, which also leads to more complex structure of the inner product of $x_{n+1}$, so that the discrete semimartingale convergence theorem is still valid for this case.

Now we give the main result of almost sure stability of the SSBE approximate solution $x_{n}$.

Theorem 5. Suppose that conditions of Lemma 3 are satisfied and the drift coefficient $f$ satisfies the linear growth condition; namely, there exists a constant $K>0$ such that

$$
|f(x, y)|^{2} \leq K\left(|x|^{2}+|y|^{2}\right)
$$

for all $x, y \in \mathbb{R}^{n}$ and $t \geq 0$. Then there exists an $h_{0}$ such that if $h<h_{0}$, the SSBE approximate solution $x_{n}$ is almost surely exponentially stable. 
Proof. Note that

$$
\begin{aligned}
\left|x_{n+1}\right|^{2}= & \left|x_{n}\right|^{2}+2 h x_{n}^{T} f\left(x_{n}^{*}, \tilde{x}_{n}\right)+\left|h f\left(x_{n}^{*}, \tilde{x}_{n}\right)\right|^{2} \\
& +\left|g\left(x_{n}^{*}, \tilde{x}_{n}\right)\right|\left(\Delta w_{n}\right)^{2} \\
& +2\left\langle x_{n}+h f\left(x_{n}^{*}, \tilde{x}_{n}\right), g\left(x_{n}^{*}, \tilde{x}_{n}\right) \Delta w_{n}\right\rangle
\end{aligned}
$$

from the SSBE method (2a) and (2b).

By using (6) and (7), we have

$$
\begin{aligned}
x_{n}^{T} f\left(x_{n}^{*}, \tilde{x}_{n}\right)= & \left(x_{n}^{T}-x_{n}^{* T}\right) f\left(x_{n}^{*}, \tilde{x}_{n}\right)+x_{n}^{* T} f\left(x_{n}^{*}, \tilde{x}_{n}\right) \\
\leq & h\left|f\left(x_{n}^{*}, \tilde{x}_{n}\right)\right|^{2}+x_{n}^{* T} f\left(x_{n}^{*}, 0\right) \\
& +x_{n}^{* T}\left(f\left(x_{n}^{*}, \tilde{x}_{n}\right)-f\left(x_{n}^{*}, 0\right)\right) \\
\leq & h\left|f\left(x_{n}^{*}, \tilde{x}_{n}\right)\right|^{2}-\frac{\lambda_{1}}{2}\left|x_{n}^{*}\right|^{2} \\
& +\left|x_{n}^{*}\right|\left|f\left(x_{n}^{*}, \tilde{x}_{n}\right)-f\left(x_{n}^{*}, 0\right)\right| .
\end{aligned}
$$

Equation (13), together with (14), shows that

$$
\begin{aligned}
\left|x_{n+1}\right|^{2} \leq & \left|x_{n}\right|^{2}+3 h^{2}\left|f\left(x_{n}^{*}, \tilde{x}_{n}\right)\right|^{2} \\
& -\lambda_{1} h\left|x_{n}^{*}\right|^{2}+2 \lambda_{2} h\left|x_{n}^{*}\right|\left|\tilde{x}_{n}\right|+\left|g\left(x_{n}^{*}, \tilde{x}_{n}\right)\right|\left(\Delta w_{n}\right)^{2} \\
& +2\left\langle x_{n}+h f\left(x_{n}^{*}, \tilde{x}_{n}\right), g\left(x_{n}^{*}, \tilde{x}_{n}\right) \Delta w_{n}\right\rangle .
\end{aligned}
$$

Therefore, by conditions (8) and (12), we have

$$
\begin{aligned}
\left|x_{n+1}\right|^{2} \leq & \left|x_{n}\right|^{2}+3 h^{2}\left(K\left|x_{n}^{*}\right|^{2}+K\left|\tilde{x}_{n}\right|^{2}\right) \\
& -\lambda_{1} h\left|x_{n}^{*}\right|^{2}+2 \lambda_{2} h\left|x_{n}^{*}\right|\left|\tilde{x}_{n}\right| \\
& +\lambda_{3}\left(\Delta w_{n}\right)^{2}\left|x_{n}^{*}\right|^{2}+\lambda_{4}\left(\Delta w_{n}\right)^{2}\left|\tilde{x}_{n}\right|^{2} \\
& +2\left\langle x_{n}+h f\left(x_{n}^{*}, \tilde{x}_{n}\right), g\left(x_{n}^{*}, \tilde{x}_{n}\right) \Delta w_{n}\right\rangle \\
\leq & \left|x_{n}\right|^{2}+\left(3 K h^{2}-\lambda_{1} h+\lambda_{2} h+\lambda_{3}\left(\Delta w_{n}\right)^{2}\right)\left|x_{n}^{*}\right|^{2} \\
& +\left(3 K h^{2}+\lambda_{2} h+\lambda_{4}\left(\Delta w_{n}\right)^{2}\right)\left|\tilde{x}_{n}\right|^{2} \\
& +2\left\langle x_{n}+h f\left(x_{n}^{*}, \tilde{x}_{n}\right), g\left(x_{n}^{*}, \tilde{x}_{n}\right) \Delta w_{n}\right\rangle .
\end{aligned}
$$

Similarly, under conditions (6), (7), and (12),

$$
\begin{aligned}
\left|x_{n}^{*}\right|^{2}= & \left\langle x_{n}+h f\left(x_{n}^{*}, \tilde{x}_{n}\right), x_{n}+h f\left(x_{n}^{*}, \tilde{x}_{n}\right)\right\rangle \\
= & \left|x_{n}\right|^{2}+2 h x_{n}^{T} f\left(x_{n}^{*}, \tilde{x}_{n}\right)+\left|h f\left(x_{n}^{*}, \tilde{x}_{n}\right)\right|^{2} \\
\leq & \left|x_{n}\right|^{2}+3 h^{2}\left|f\left(x_{n}^{*}, \tilde{x}_{n}\right)\right|^{2}-\lambda_{1} h\left|x_{n}^{*}\right|^{2}+2 \lambda_{2} h\left|x_{n}^{*}\right|\left|\tilde{x}_{n}\right| \\
\leq & \left|x_{n}\right|^{2}+3 K h^{2}\left(\left|x_{n}^{*}\right|^{2}+\left|\tilde{x}_{n}\right|^{2}\right) \\
& -\lambda_{1} h\left|x_{n}^{*}\right|^{2}+\lambda_{2} h\left(\left|x_{n}^{*}\right|^{2}+\left|\tilde{x}_{n}\right|^{2}\right),
\end{aligned}
$$

which implies that

$$
\left(1-3 K h^{2}+\lambda_{1} h-\lambda_{2} h\right)\left|x_{n}^{*}\right|^{2} \leq\left|x_{n}\right|^{2}+\left(3 K h^{2}+\lambda_{2} h\right)\left|\tilde{x}_{n}\right|^{2} \text {. }
$$

By Vieta theorem, because the discriminant of the quadratic equation $1-3 K h^{2}+\lambda_{1} h-\lambda_{2} h=0$ is positive and $-3 K<0$, there must exist an $h_{1}>0$ such that $1-3 K h^{2}+\lambda_{1} h-\lambda_{2} h>0$ for any $0<h<h_{1}$; then

$$
\begin{aligned}
\left|x_{n}^{*}\right|^{2} \leq & \frac{1}{1-3 K h^{2}+\lambda_{1} h-\lambda_{2} h}\left|x_{n}\right|^{2} \\
& +\frac{3 K h^{2}+\lambda_{2} h}{1-3 K h^{2}+\lambda_{1} h-\lambda_{2} h}\left|\tilde{x}_{n}\right|^{2} .
\end{aligned}
$$

For simplicity, in what follows, the formula $1-3 K h^{2}+\lambda_{1} h-$ $\lambda_{2} h$ is denoted by G. Combining (16) and (19) leads us to

$$
\begin{aligned}
\left|x_{n+1}\right|^{2} \leq & \left|x_{n}\right|^{2}+\frac{1-G+\lambda_{3}\left(\Delta w_{n}\right)^{2}}{G}\left|x_{n}\right|^{2} \\
& +\frac{\left(1-G+\lambda_{3}\left(\Delta w_{n}\right)^{2}\right)\left(3 K h^{2}+\lambda_{2} h\right)}{G}\left|\tilde{x}_{n}\right|^{2} \\
& +\left(3 K h^{2}+\lambda_{2} h+\lambda_{4}\left(\Delta w_{n}\right)^{2}\right)\left|\tilde{x}_{n}\right|^{2} \\
& +2\left\langle x_{n}+h f\left(x_{n}^{*}, \tilde{x}_{n}\right), g\left(x_{n}^{*}, \tilde{x}_{n}\right) \Delta w_{n}\right\rangle .
\end{aligned}
$$

For any positive constant $C>1$, we have

$$
\begin{aligned}
& C^{(i+1) h}\left|x_{i+1}\right|^{2}-C^{i h}\left|x_{i}\right|^{2} \\
& =C^{(i+1) h}\left(\left|x_{i+1}\right|^{2}-\left|x_{i}\right|^{2}\right)+\left(C^{(i+1) h}-C^{i h}\right)\left|x_{i}\right|^{2},
\end{aligned}
$$

which yields

$$
\begin{aligned}
& C^{(i+1) h}\left|x_{i+1}\right|^{2}-C^{i h}\left|x_{i}\right|^{2} \\
& \leq C^{(i+1) h}\left[1-C^{-h}+\frac{1-G+\lambda_{3}\left(\Delta w_{i}\right)^{2}}{G}\right]\left|x_{i}\right|^{2} \\
& +C^{(i+1) h}\left[\frac{\left(1-G+\lambda_{3}\left(\Delta w_{i}\right)^{2}\right)\left(3 K h^{2}+\lambda_{2} h\right)}{G}\right. \\
& \left.+3 K h^{2}+\lambda_{2} h+\lambda_{4}\left(\Delta w_{i}\right)^{2}\right]\left|\tilde{x}_{i}\right|^{2} \\
& +2 C^{(i+1) h}\left\langle x_{i}+h f\left(x_{i}^{*}, \tilde{x}_{i}\right), g\left(x_{i}^{*}, \tilde{x}_{i}\right) \Delta w_{i}\right\rangle
\end{aligned}
$$


by using (20). Summing up both sides of inequality (22) from $i=0$ to $n-1(n \geq 1)$, we get

$$
\begin{aligned}
C^{n h}\left|x_{n}\right|^{2} \leq & \left|x_{0}\right|^{2}+\left[1-C^{-h}+\frac{1-G}{G}\right] \sum_{i=0}^{n-1} C^{(i+1) h}\left|x_{i}\right|^{2} \\
& +\left(\frac{\lambda_{3}}{G}\right) \sum_{i=0}^{n-1} C^{(i+1) h}\left(\Delta w_{i}\right)^{2}\left|x_{i}\right|^{2} \\
& +\frac{3 K h^{2}+\lambda_{2} h}{G-1} \sum_{i=0}^{n-1} C^{(i+1) h}\left|\tilde{x}_{i}\right|^{2} \\
& +\left[\frac{\left(3 K h^{2}+\lambda_{2} h\right) \lambda_{3}}{G}+\lambda_{4}\right] \sum_{i=0}^{n-1} C^{(i+1) h}\left(\Delta w_{i}\right)^{2}\left|\tilde{x}_{i}\right|^{2} \\
& +2 \sum_{i=0}^{n-1} C^{(i+1) h}\left\langle x_{i}+h f\left(x_{i}^{*}, \tilde{x}_{i}\right), g\left(x_{i}^{*}, \tilde{x}_{i}\right) \Delta w_{i}\right\rangle .
\end{aligned}
$$

Let $\mathscr{M}_{n}^{(1)}=\sum_{i=0}^{n-1} C^{(i+1) h}\left|x_{i}\right|^{2}\left(\left(\Delta w_{i}\right)^{2}-h\right)$. Since $E\left(\left(\Delta w_{n}\right)^{2}-\right.$ $h)=0$ and $x_{n}$ is $\mathscr{F}_{n h}$-measurable, we obtain

$$
\begin{aligned}
E & {\left[\mathscr{M}_{n}^{(1)} \mid \mathscr{F}_{(n-1) h}\right] } \\
& =\mathscr{M}_{n-1}^{(1)}+E\left[C^{n h}\left|x_{n-1}\right|^{2}\left(\left(\Delta w_{n-1}\right)^{2}-h\right) \mid \mathscr{F}_{(n-1) h}\right] \\
& =\mathscr{M}_{n-1}^{(1)}+C^{n h}\left|x_{n-1}\right|^{2} E\left[\left(\left(\Delta w_{n-1}\right)^{2}-h\right) \mid \mathscr{F}_{(n-1) h}\right] \\
& =\mathscr{M}_{n-1}^{(1)},
\end{aligned}
$$

which implies that $\mathscr{M}_{n}^{(1)}$ is a martingale.

Similarly,

$$
\begin{gathered}
\mathscr{M}_{n}^{(2)}=\sum_{i=0}^{n-1} C^{(i+1) h}\left|\tilde{x}_{i}\right|^{2}\left(\left(\Delta w_{i}\right)^{2}-h\right), \\
\mathscr{M}_{n}^{(3)}=2 \sum_{i=0}^{n-1} C^{(i+1) h}\left\langle x_{i}+h f\left(x_{i}^{*}, \tilde{x}_{i}\right), g\left(x_{i}^{*}, \tilde{x}_{i}\right) \Delta w_{i}\right\rangle
\end{gathered}
$$

are also martingales. Therefore,

$$
\mathscr{M}_{n}=\frac{\lambda_{3}}{G} \mathscr{M}_{n}^{(1)}+\left[\frac{\lambda_{3}\left(3 K h^{2}+\lambda_{2} h\right)}{G}+\lambda_{4}\right] \mathscr{M}_{n}^{(2)}+\mathscr{M}_{n}^{(3)}
$$

is a martingale with $\mathscr{M}_{0}=0$. Then we have

$$
\begin{aligned}
C^{n h}\left|x_{n}\right|^{2} \leq & \left|x_{0}\right|^{2}+\left[-C^{-h}+\frac{1+\lambda_{3} h}{G}\right] \sum_{i=0}^{n-1} C^{(i+1) h}\left|x_{i}\right|^{2} \\
& +\left[\frac{\left(1+\lambda_{3} h\right)\left(3 K h^{2}+\lambda_{2} h\right)}{G}+\lambda_{4} h\right] \\
& \times \sum_{i=0}^{n-1} C^{(i+1) h}\left|\tilde{x}_{i}\right|^{2}+\mathscr{M}_{n} .
\end{aligned}
$$

Noting that there are two approximating cases of the time dependent delay term $X\left(t_{n}-\tau\left(t_{n}\right)\right)$ in (3), the following analysis will be divided into two situations. First, we have

$$
\begin{aligned}
C^{n h}\left|x_{n}\right|^{2} \leq & \left|x_{0}\right|^{2}+\left[-C^{-h}+\frac{1+\lambda_{3} h}{G}\right] \sum_{i=0}^{n-1} C^{(i+1) h}\left|x_{i}\right|^{2} \\
& +\left[\frac{\left(1+\lambda_{3} h\right)\left(3 K h^{2}+\lambda_{2} h\right)}{G}+\lambda_{4} h\right] \\
& \times \sum_{i=0}^{n-1} C^{(i+1) h}\left|\psi\left(t_{i}-\tau\left(t_{i}\right)\right)\right|^{2}+\mathscr{M}_{n}
\end{aligned}
$$

under condition $t_{n}<\tau\left(t_{n}\right)$. There exists an $h_{2}$ such that, for any $0<h<h_{1} \wedge h_{2}, C^{-h}-\left(1+\lambda_{3} h\right) / G>0$, where $\wedge$ is the minimal operator. Further, we set $A_{n}^{1}=0$ for any nonnegative integer $n, A_{0}^{2}=0$,

$$
A_{n}^{2}=\left[C^{-h}-\frac{1+\lambda_{3} h}{G}\right] \sum_{i=0}^{n-1} C^{(i+1) h}\left|x_{i}\right|^{2}
$$

for $n \geq 1$, and

$$
\begin{gathered}
Z_{n}=C^{n h}\left|x_{n}\right|^{2}, \\
Z_{0}=\left|x_{0}\right|^{2}+\left[\frac{\left(1+\lambda_{3} h\right)\left(3 K h^{2}+\lambda_{2} h\right)}{G}+\lambda_{4} h\right] \\
\times \sum_{i=0}^{n-1} C^{(i+1) h}\left|\psi\left(t_{i}-\tau\left(t_{i}\right)\right)\right|^{2} .
\end{gathered}
$$

Therefore, a direct application of Theorem 4 to the sequence $Z_{n}$ yields that

$$
\limsup _{n \rightarrow \infty} C^{n h}\left|x_{n}\right|^{2} \leq+\infty .
$$

Choose the $\gamma>0$, such that $C=e^{\gamma}$ and hence

$$
\limsup _{n \rightarrow \infty} e^{\gamma n h}\left|x_{n}\right|^{2} \leq+\infty \text {. }
$$

We therefore obtain that, for any $0<h<h_{1} \wedge h_{2}$,

$$
\limsup _{n \rightarrow \infty} \frac{1}{n h} \log \left|x_{n}\right| \leq-\frac{\gamma}{2}, \quad \text { a.s }
$$

as required.

Now, let us discuss the second situation: $t_{n} \geq \tau\left(t_{n}\right)$. Inequality (27) gives

$$
\begin{aligned}
C^{n h}\left|x_{n}\right|^{2} \leq & \left|x_{0}\right|^{2}+\left[-C^{-h}+\frac{1+\lambda_{3} h}{G}\right] \sum_{i=0}^{n-1} C^{(i+1) h}\left|x_{i}\right|^{2} \\
& +2\left[\frac{\left(1+\lambda_{3} h\right)\left(3 K h^{2}+\lambda_{2} h\right)}{G}+\lambda_{4} h\right] \\
& \times \sum_{i=0}^{n-1} C^{(i+1) h}\left(\mu^{2}\left|x_{i-q_{i}+1}\right|^{2}+(1-\mu)^{2}\left|x_{i-q_{i}}\right|^{2}\right) \\
& +\mathscr{M}_{n} .
\end{aligned}
$$




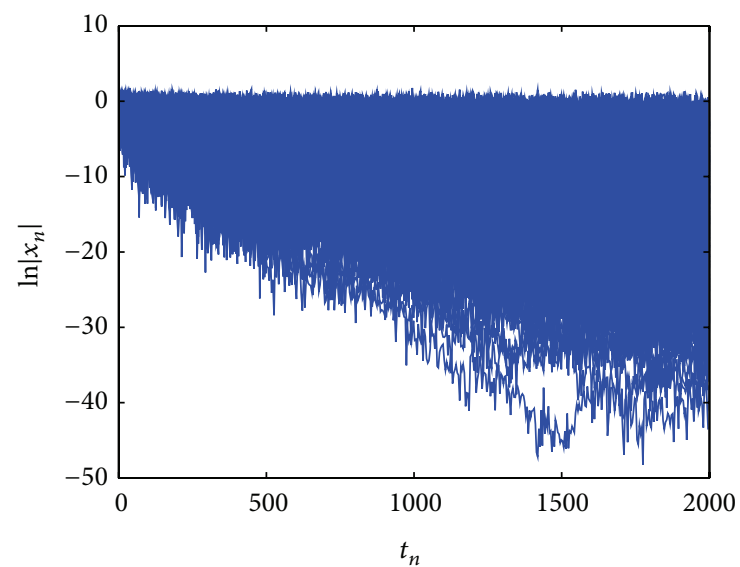

(a) $h=0.4 ; \mu=0.0$

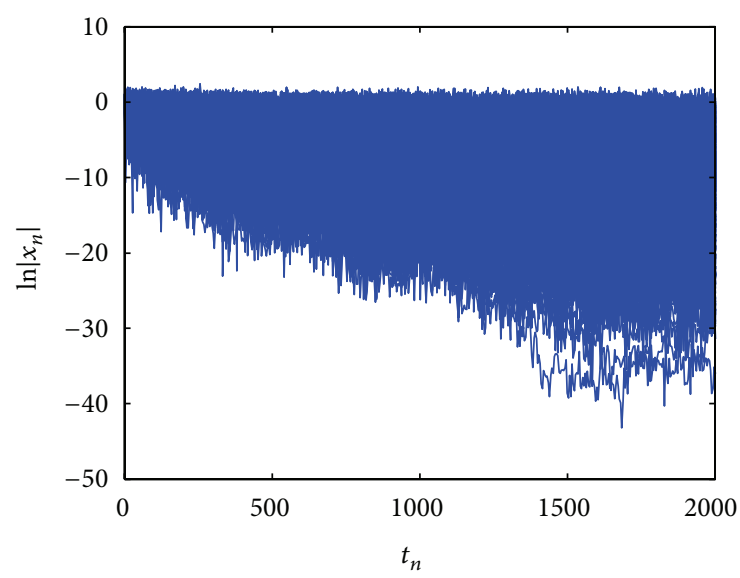

(c) $h=0.4 ; \mu=1.0$

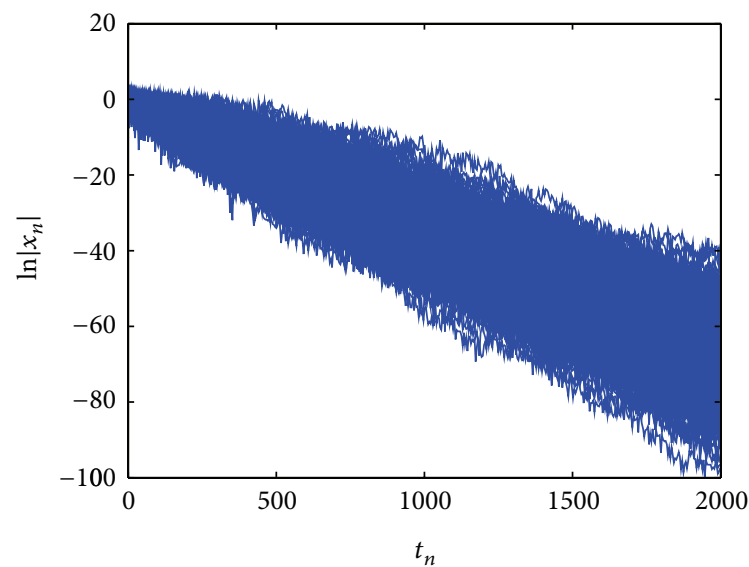

(b) $h=0.4 ; \mu=0.5$

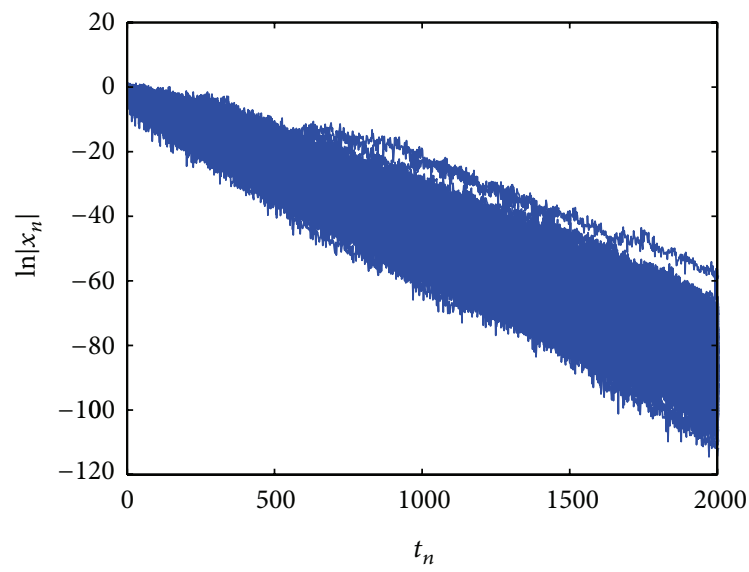

(d) $h=0.2 ; \mu=1.0$

FIGURE 1: Almost sure stability of SSBE method applied to (1) with $\alpha=-10, \sigma=1, \lambda=1$, and $\beta=2$.

Since

$$
\begin{aligned}
& \sum_{i=0}^{n-1} C^{(i+1) h}\left|x_{i-q_{i}}\right|^{2} \\
& =\sum_{i=-q_{i}}^{-1} C^{\left(i+q_{i}+1\right) h}\left|x_{i}\right|^{2}+\sum_{i=0}^{n-1} C^{\left(i+q_{i}+1\right) h}\left|x_{i}\right|^{2}-\sum_{i=n-q_{i}}^{n-1} C^{\left(i+q_{i}+1\right) h}\left|x_{i}\right|^{2}, \\
& \sum_{i=0}^{n-1} C^{(i+1) h}\left|x_{i-q_{i}+1}\right|^{2} \\
& =\sum_{i=-q_{i}+1} C^{\left(i+q_{i}\right) h}\left|x_{i}\right|^{2}+\sum_{i=0}^{n-1} C^{\left(i+q_{i}\right) h}\left|x_{i}\right|^{2}-\sum_{i=n-q_{i}+1}^{n-1} C^{\left(i+q_{i}\right) h}\left|x_{i}\right|^{2},
\end{aligned}
$$

we have

$$
Z_{n} \leq Z_{0}-A_{n}^{2}+\mathscr{M}_{n}
$$

where

$$
\begin{aligned}
Z_{n}= & C^{n h}\left|x_{n}\right|^{2}+2 \mu^{2}\left[\frac{\left(1+\lambda_{3} h\right)\left(3 K h^{2}+\lambda_{2} h\right)}{G}+\lambda_{4} h\right] \\
& \times \sum_{i=n-q_{i}+1}^{n-1} C^{\left(i+q_{i}\right) h}\left|x_{i}\right|^{2} \\
& +2(1-\mu)^{2}\left[\frac{\left(1+\lambda_{3} h\right)\left(3 K h^{2}+\lambda_{2} h\right)}{G}+\lambda_{4} h\right] \\
& \times \sum_{i=n-q_{i}}^{n-1} C^{\left(i+q_{i}+1\right) h}\left|x_{i}\right|^{2}, \\
A_{n}^{2}= & {\left[C^{-h}-\frac{1+\lambda_{3} h}{G}-2 \mu^{2}\right.}
\end{aligned}
$$

$$
\times\left(\frac{\left(1+\lambda_{3} h\right)\left(3 K h^{2}+\lambda_{2} h\right)}{G}+\lambda_{4} h\right) C^{q_{i}-1}
$$




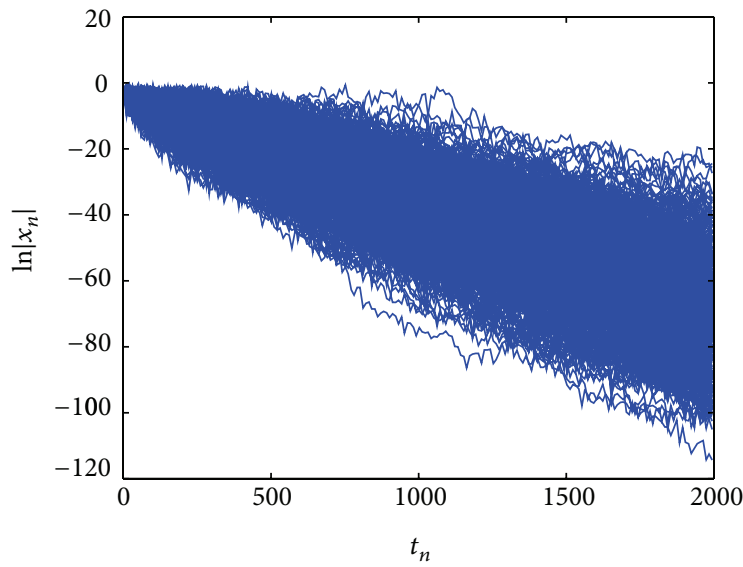

(a) $h=1.0 ; \mu=0.0$

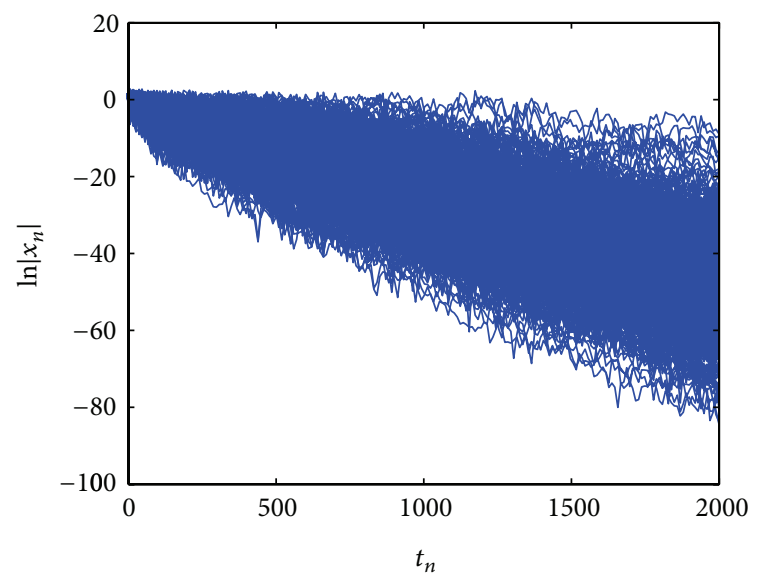

(c) $h=1.0 ; \mu=1.0$

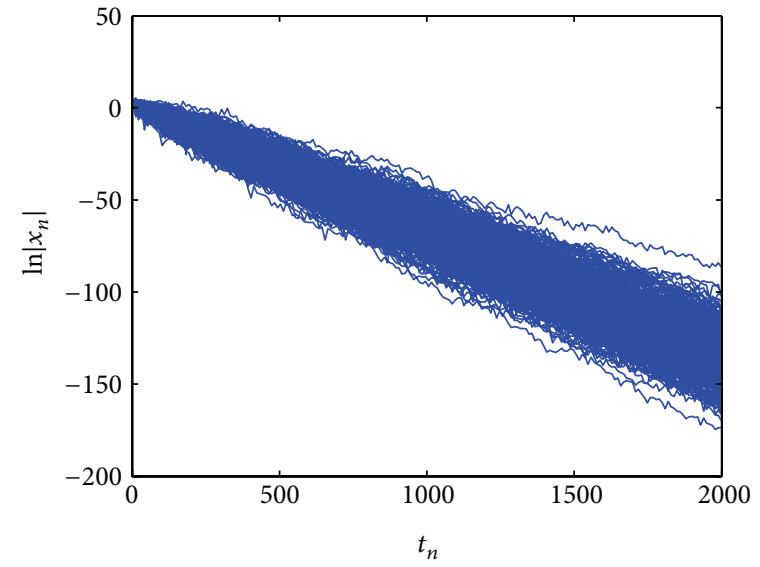

(b) $h=1.0 ; \mu=0.5$



(d) $h=0.9 ; \mu=1.0$

FIGURE 2: Almost sure stability of SSBE method applied to (1) with $\alpha=-10, \sigma=1, \lambda=3$, and $\beta=1$.

$$
\begin{aligned}
& \left.-2(1-\mu)^{2}\left(\frac{\left(1+\lambda_{3} h\right)\left(3 K h^{2}+\lambda_{2} h\right)}{G}+\lambda_{4} h\right) C^{q_{i}}\right] \\
& \times \sum_{i=0}^{n-1} C^{(i+1) h}\left|x_{i}\right|^{2} .
\end{aligned}
$$

There exists an $h_{3}$ such that, for any $0<h<h_{1} \wedge h_{3}$,

$$
\begin{aligned}
C^{-h}- & \frac{1+\lambda_{3} h}{G}-2 \mu^{2}\left(\frac{\left(1+\lambda_{3} h\right)\left(3 K h^{2}+\lambda_{2} h\right)}{G}+\lambda_{4} h\right) C^{q_{i}-1} \\
& -2(1-\mu)^{2}\left(\frac{\left(1+\lambda_{3} h\right)\left(3 K h^{2}+\lambda_{2} h\right)}{G}+\lambda_{4} h\right) C^{q_{i}}>0 .
\end{aligned}
$$

Similarly, the solution $x_{n}$ is almost surely exponentially stable by using Theorem 4 .

Consequently, we conclude that, for any $0<h<h_{1} \wedge$ $h_{2} \wedge h_{3}$, the SSBE approximate solution $x_{n}$ is almost surely exponentially stable.

\section{Numerical Experiments}

In this section, we present some numerical examples to illustrate our theoretical analysis. We calculated 500 sample paths of the approximate solution and plotted them along the time $t$ (see, e.g., Figure 1(a)). Figures 1 to 2 depict the results by SSBE method in the log-scaled vertical axis. Here we set $d=1$, drift coefficient $f=\alpha X(t)+\sigma \sin (X(t-\tau))$, diffusion coefficient $g=\lambda X(t)+\beta \sin (X(t-\tau))$, initial function $\psi(t)=$ $t+1$, and delay function $\tau(t)=3(\cos (t / 2))^{2}+3$.

Figures 1 to 2 show that the SSBE approximate solution $x_{n}$ has better almost sure stability in the case of choosing the parameter $\mu=0.5$ in (3). Comparing Figures $1(\mathrm{c})$ and 1(d), the almost sure stability of approximate solution can be obtained by reducing the step size $h$.

\section{Conflict of Interests}

The authors declare that there is no conflict of interests regarding the publication of this paper.

\section{Acknowledgments}

This work was partially supported by E-Institutes of Shanghai Municipal Education Commission (no. E03004), Natural 
Science Foundation of Shanghai (no. 14ZR1431300), and Innovation Program of Shanghai Municipal Education Commission (no. 14YZ078).

\section{References}

[1] Y. Saito and T. Mitsui, "Stability analysis of numerical schemes for stochastic differential equations," SIAM Journal on Numerical Analysis, vol. 33, no. 6, pp. 2254-2267, 1996.

[2] W. Cao, M. Liu, and Z. Fan, "MS-stability of the EulerMaruyama method for stochastic differential delay equations," Applied Mathematics and Computation, vol. 159, no. 1, pp. 127135, 2004.

[3] C. Huang, "Mean square stability and dissipativity of two classes of theta methods for systems of stochastic delay differential equations," Journal of Computational and Applied Mathematics, vol. 259, pp. 77-86, 2014.

[4] A. Rodkina and H. Schurz, "Almost sure asymptotic stability of drift-implicit $\theta$-methods for bilinear ordinary stochastic differential equations in $R^{1}$," Journal of Computational and Applied Mathematics, vol. 180, no. 1, pp. 13-31, 2005.

[5] D. J. Higham, X. Mao, and C. Yuan, "Almost sure and moment exponential stability in the numerical simulation of stochastic differential equations," SIAM Journal on Numerical Analysis, vol. 45, no. 2, pp. 592-609, 2007.

[6] S. Pang, F. Deng, and X. Mao, "Almost sure and moment exponential stability of Euler-Maruyama discretizations for hybrid stochastic differential equations," Journal of Computational and Applied Mathematics, vol. 213, no. 1, pp. 127-141, 2008.

[7] A. Rodkina, H. Schurz, and L. Shaikhet, "Almost sure stability of some stochastic dynamical systems with memory," Discrete and Continuous Dynamical Systems Series A, vol. 21, no. 2, pp. 571-593, 2008.

[8] F. Wu, X. Mao, and L. Szpruch, "Almost sure exponential stability of numerical solutions for stochastic delay differential equations," Numerische Mathematik, vol. 115, no. 4, pp. 681-697, 2010.

[9] H. Schurz, "Almost sure asymptotic stabilit y and convergence of stochastic theta methods applied to system of linear SDEs in $\mathbb{R}^{d}$, , Random Operators and Stochastic Equations, vol. 19, pp. 111129, 2011.

[10] F. Wu, X. Mao, and P. E. Kloeden, "Almost sure exponential stability of the Euler-Maruyama approximations for stochastic functional differential equations," Random Operators and Stochastic Equations, vol. 19, no. 2, pp. 165-186, 2011.

[11] X. Mao and L. Szpruch, "Strong convergence and stability of implicit numerical methods for stochastic differential equations with non-globally Lipschitz continuous coefficients," Journal of Computational and Applied Mathematics, vol. 238, pp. 14-28, 2013.

[12] X. Mao and S. Sabanis, "Numerical solutions of stochastic differential delay equations under local Lipschitz condition," Journal of Computational and Applied Mathematics, vol. 151, no. 1, pp. 215-227, 2003.

[13] X. J. Wang and S. Q. Gan, "The improved split-step backward Euler method for stochastic differential delay equations," International Journal of Computer Mathematics, vol. 88, no. 11, pp. 2359-2378, 2011.

[14] Q. Guo and X. Tao, "Almost sure and $L^{P}$ con vergence of splitstep backward Euler method for stochastic delay differential equation," Abstract and Applied Analysis, vol. 2014, Article ID 390418, 7 pages, 2014.
[15] X. Mao, Stochastic Differential Equations and Their Applications, Horwood, Chichester, UK, 1997. 


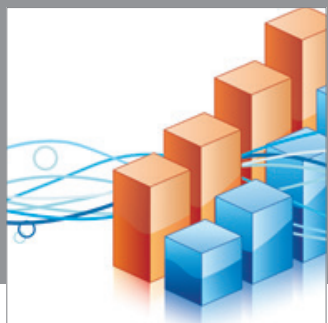

Advances in

Operations Research

mansans

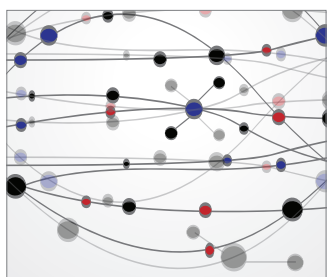

The Scientific World Journal
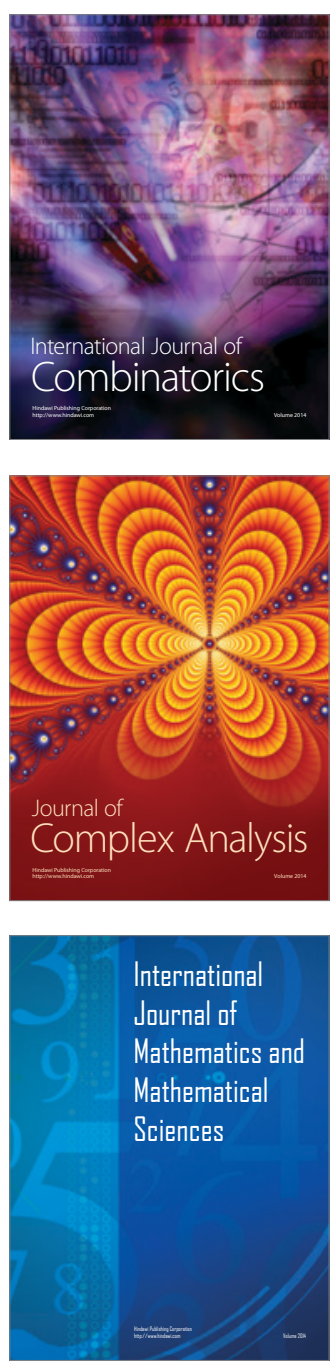
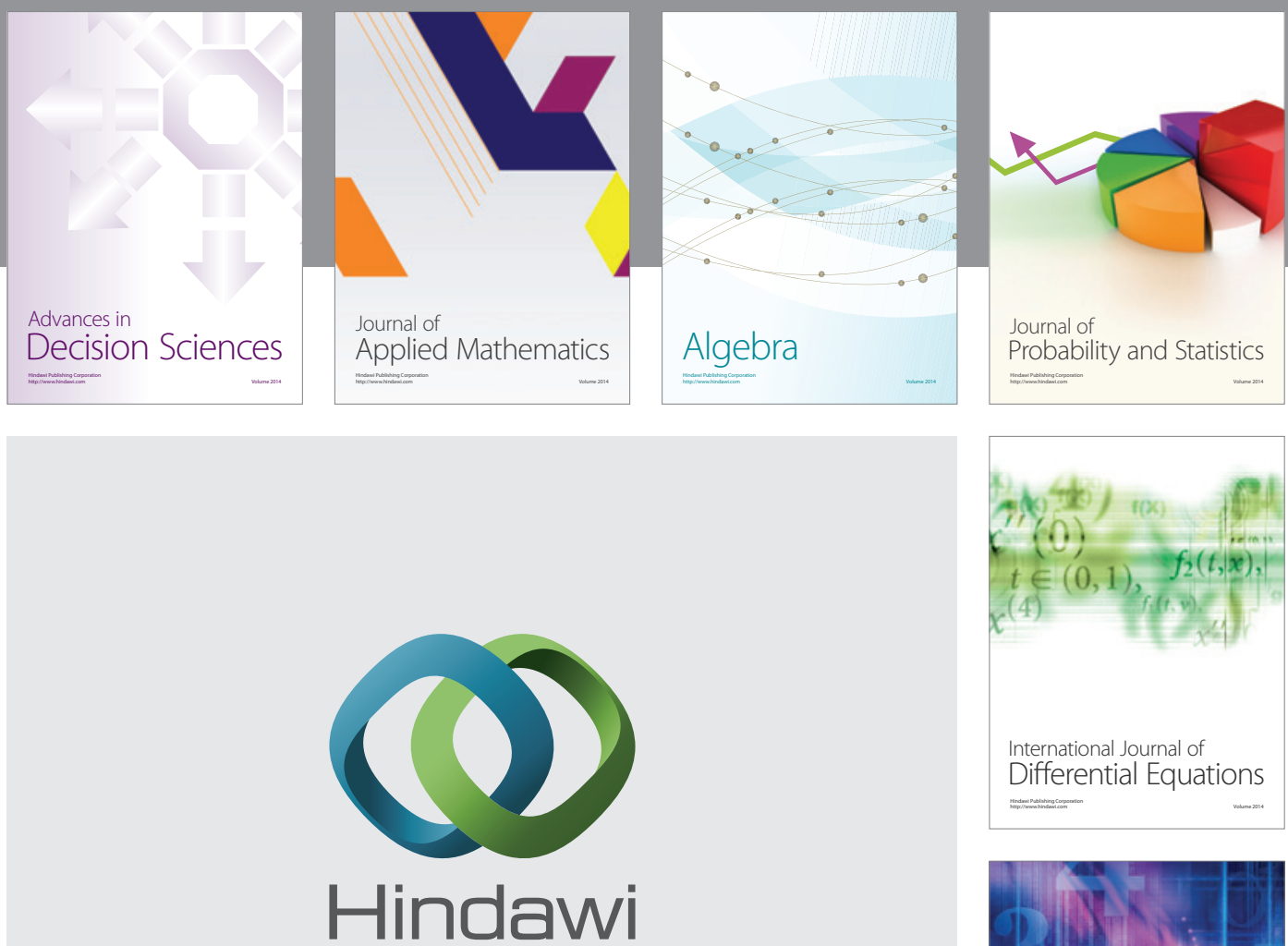

Submit your manuscripts at http://www.hindawi.com
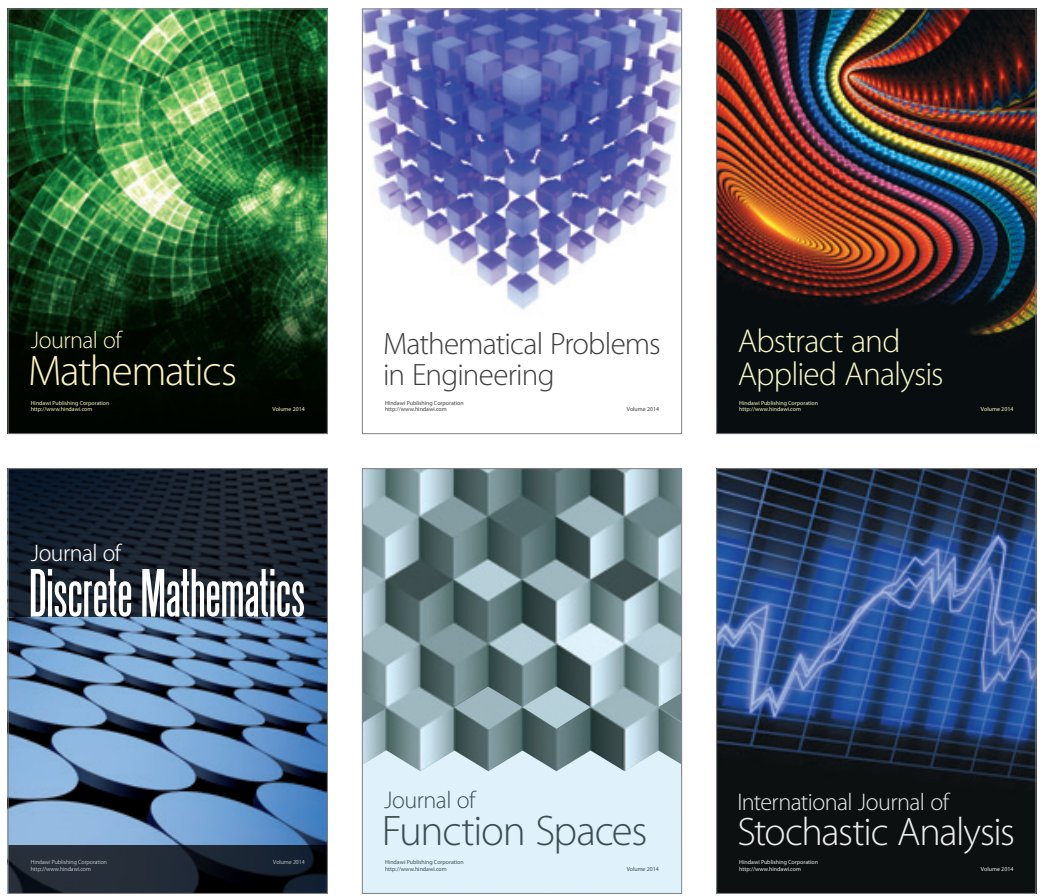

Journal of

Function Spaces

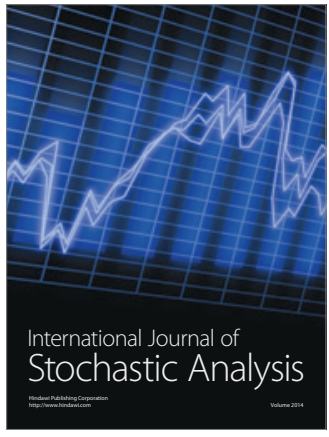

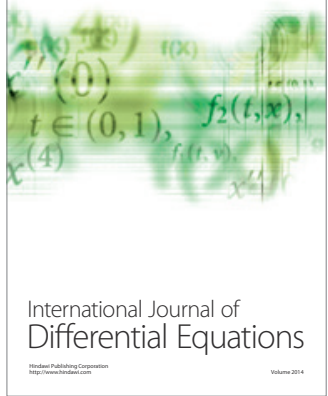
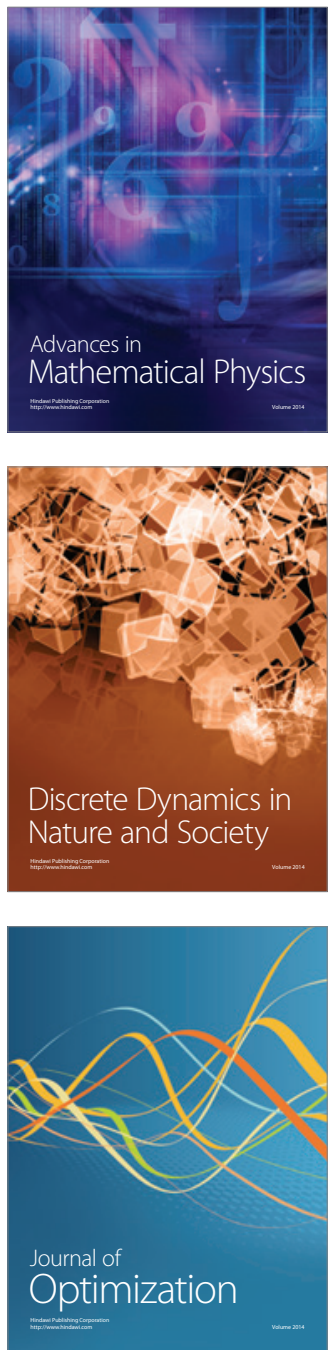